Danksagung. Für die Durchführung der kompetenten bildgebenden Diagnostik und die Bereitstellung der Bilddokumente sind die Autoren den Kollegen der Klinik für Radiologie und Nuklearmedizin, Universitätsklinikum Magdeburg A. ö. R., sehr zu Dank verpflichtet.

\section{Einhaltung ethischer Richtlinien}

Interessenkonflikt. Z. Halloul, M. Mhanna, U. Barth, A. Udelnow und F. Meyer geben an, dass kein Interessenkonflikt besteht.

Dieser Beitrag beinhaltet keine Studien an Menschen oder Tieren.

\section{Literatur}

1. Halloul Z, Meyer F, Grote R et al (2005) Selective embolization of splenic artery aneurysm - case report. Eur Surg 37(1):59-62

2. Meyer A, Uder M, Lang W et al (2010) Aneurysmen an viszeralen Arterien. Zentralbl Chir 135(5):416420

3. Ambrosetti P, Meyer P, Mentha G et al (1987) Aneurismes des arteres pancreatico-duodenales et hepatiques. Chirurgie 113:46-56

4. Croner RS, Anders K, Uder M et al (2006) Aneurysmen viszeraler Arterien. Dtsch Ärztebl 103(20): A1367-A1371

5. Schmidt G, Görg C (2008) Kursbuch Ultraschall (ISBN 9783131191052). Georg Thieme Verlag KG, Stuttgart

6. Michels NA (1951) The hepatic, cystic and retroduodenal arteries and their relations to the biliary ducts with samples of the entire celiacal blood supply. Ann Surg 133(4):503-524

7. Bueschel P, Meyer F, Weber M et al (2013) Rare aneurysm of the hepatic artery with overlap to the gastroduodenal artery in very uncommon coincidence with occurence of hepatomesenteric trunk - first case reported in the literature. Wien Klin Wochenschr 125(3-4):111-114

8. Carr SC, Mahvi DM, Hoch JR et al (2001) Visceral artery aneurysm rupture. J Vasc Surg 33:806-811

9. Tulsyan N, Kashyap VS, Greenberg R (2007) The endovascular management of visceral artery aneurysms and pseudoaneurysms. J Vasc Surg 45:276283 (discussion 283)

10. Balderi A, Antonietti A, Pedrazzini F et al (2010) Management of hepatic artery aneurysm by endovascular exclusion using multilayer cardiatis stent. Cardiovasc Intervent Radiol 33:1282-1286

Gefässchirurgie 2015 • 20:49-49 DOI 10.1007/s00772-014-1445-4

Online publiziert: 28. Januar 2015 ○ Springer-Verlag Berlin Heidelberg 2015

T. Schmitz-Rixen ${ }^{1}$ - F. Adili ${ }^{2} \cdot$ R. Bauersachs ${ }^{3} \cdot$ I. Eichler ${ }^{4}$. J. Hoffmann ${ }^{5}$. J. Klocker ${ }^{6}$. J. Kobba ${ }^{7} \cdot$ T. Petzold $^{8} \cdot$ H. Wenk ${ }^{9} \cdot$ R.T. Grundmann ${ }^{10}$

${ }^{1}$ Klinik für Gefäß- und Endovascularchirurgie, Klinikum der Goethe-Universität Frankfurt am Main

2 Klinik für Gefäß- und Endovascularchirurgie, Klinikum Darmstadt

${ }^{3}$ Klinik für Gefäßmedizin, Angiologie, Klinikum Darmstadt

${ }^{4}$ Klinik für Herzchirurgie, Klinikum Dortmund

${ }^{5}$ Sektion Gefäßchirurgie, Klinik für Allgemein-, Viszeral- und

Transplantationschirurgie, Universitätsklinikum Essen

${ }^{6}$ Universitätsklinik für Gefäßchirurgie, Innsbruck

${ }^{7}$ Thorax, Herz- und Gefäßchirurgie, Universitätsklinikum Tübingen

${ }^{8}$ Klinik für Gefäß- und endovaskuläre Chirurgie, Klinikum Saarbrücken

${ }^{9}$ Allgemein-, Visceralchirurgie und Gefäßchirurgie, Klinikum Bremen-Nord

${ }^{10}$ DIGG der Deutschen Gesellschaft für Gefäßchirurgie und Gefäßmedizin, Berlin

\title{
Erratum zu: Antikoagulation mit Argatroban bei vaskulären und endovaskulären Operationen und Interventionen und Verdacht auf heparininduzierte Thrombozytopenie Typ II (HIT II)
}

In diesem Beitrag wurde auf S.1 eine falsche Thrombozytenzahl angegeben. Statt 150.00/ $\mu$ l muss es heißen:

Die Thrombozytopenie ist bei 85-90\% der Patienten das erste Anzeichen einer HIT II, mit einem abrupten Abfall der Thrombozyten unter $150.000 / \mu$ l oder um mehr als $50 \%$ des Basiswertes.

Wir bitten, die korrekte Nennung zu beachten und den Fehler zu entschuldigen.

Die Redaktion

\section{Korrespondenzadresse}

\section{T. Schmitz-Rixen}

Klinik für Gefäß- und Endovascularchirurgie, Klinikum der Goethe-Universität Frankfurt am Main

Theodor-Stern-Kai 7, 60590 Frankfurt am Main schmitz-rixen@em.uni-frankfurt.de
Die Online-Version des Originalartikels können Sie unter http://dx.doi.org/10.1007/s00772-014-1413-z finden. 